\title{
Light dark matter from inflaton decay
}

\section{Takeo Moroi and Wen Yin}

Department of Physics, The University of Tokyo,

Tokyo 113-0033, Japan

E-mail: moroi@phys.s.u-tokyo.ac.jp, yinwen@tuhep.phys.tohoku.ac.jp

ABSTRACT: We propose a simple mechanism of light dark matter (DM) production from the decay of the oscillating inflaton condensation. If the reheating temperature after inflation is higher than the inflaton mass, which is of the same order of the momentum of the DM at the time of the production, the DM momentum can be suppressed compared to the temperature of the thermal plasma if the interaction of the DM is weak enough. Consequently, the DM can be cold enough to avoid the observational constraints on the warm DM, like the Lyman- $\alpha$ bound even if the DM mass is small. We study the bosonic and fermionic DM production from the inflaton decay, taking into account the effect of the stimulated emission and Pauli blocking, respectively. In both cases, the DM can be cold and abundant enough to be a viable candidate of the DM. We also apply our mechanism to the production of isocurvature-problem-free axion DM and Dirac sea DM of right-handed neutrino consistent the seesaw relation for the active neutrino masses.

KEywords: Beyond Standard Model, Cosmology of Theories beyond the SM

ARXiv EPrint: 2011.09475 


\section{Contents}

1 Introduction 1

2 Basic formalisms 2

3 Light DM from inflaton decay $\quad \mathbf{5}$

3.1 Cold DM from inflaton decay 5

$\begin{array}{ll}3.2 & \text { DM production in a narrow resonance regime }\end{array}$

4 DM models $\quad 11$

$\begin{array}{lll}\text { 4.1 DASER production of isocurvature free axion DM } & 11\end{array}$

$\begin{array}{lll}4.2 & \text { Dirac sea DM of right handed neutrino } & 13\end{array}$

5 Reheating from evaporating inflaton $\quad 16$

6 Conclusions and discussion $\quad 20$

\section{Introduction}

A long-standing puzzle of particle physics and cosmology is the origin of dark matter (DM). The DM is known to be (very) weakly coupled to the standard model (SM) particles, stable, cold, and abundant in the present Universe. However the mass, the interactions, and the production mechanism are still not clear.

The DM mass may be small, in which case the stability (or longevity) is easily explained due to the kinematics (or suppressed decay width). From the point of view of DM direct detections, if the mass is small enough, the recoil energy of a nucleon via the DM-nucleon scattering is highly suppressed, which is consistent with the null result of the direct detection experiments for WIMP. Nevertheless a light DM can be searched for from different approaches in the near future (e.g. ref. [1] for review of axion searches, refs. [2-4] for direct detection with electron recoils).

A difficulty of the light DM is the production in the early Universe. If it were produced thermally, like the WIMP, the number density cannot be larger than that of the SM photons, and may be too small unless the mass is larger than $\mathrm{eV}$. Moreover, it may be too hot to be consistent with structure formation. For instance a thermal relic of sterile neutrino has to be heavier than $2-5 \mathrm{keV}[5,6]$ from Lyman- $\alpha$ forest data. If it is produced from freeze-in the bound is even severer [7].

The production of light DM has been discussed widely. An axion/axion-like particle (ALP) can be produced via the misalignment mechanism [8-10]. A light hidden photon DM can be produced gravitationally $[11,12]$. (Gravitational effect during inflation is also important for the axion/ALP DM $[8-10,13,14])$. The hidden photon DM can be produced 
from a parametric/tachyonic resonance by coupling to the QCD axion [15-18] (see also ref. [19]). In addition, the axion (or ALP) production from the parametric resonance of another scalar field was discussed [20,21]. A light DM may be produced by the decays of heavy particles at an early epoch [22].

In this paper, we study a new possibility of light DM production. The light DM is produced from the decay of an inflaton which reheats the Universe. ${ }^{1}$ We show that, if the reheating temperature $T_{R}$ is higher than the inflaton mass $m_{\phi}$, the DM particles from the inflaton decays can become cold enough due to the red shift. If the inflaton coupling to the SM particles and the DM is not too small, $T_{R} \gtrsim m_{\phi}$ can be realized due to the dissipation effect [23-29]. In particular, for the case that the DM is bosonic, the DM production can be enhanced due to the stimulated emission effect like LASER (light amplification by stimulated emission of radiation); we call such a mechanism as DASER. In addition, if the DM is fermionic, a Dirac sea DM can be realized. Interestingly, the produced DM does not suffer from the isocurvature problem. Axion(-like) DM and Dirac sea DM of a right-handed neutrino, which is responsible to the neutrino oscillations, are discussed.

Heavy DM production in association with the reheating was studied, e.g. due to the decay (with a late time annihilation) of the inflaton [30-34], during thermalization at the last stage of the reheating [35-38] and during the preheating [39, 40]. In contrast, we focus on general "light" DM production, for which we find that $T_{R} / m_{\phi} \gtrsim 1$ is important. In ref. [21], the QCD axion production from parametric resonance of the Peccei-Quinn (PQ) field was considered, however the PQ field condensate does not dominate and reheat the Universe.

This paper is organized as follows. In section 2, we present the basic formalisms used in our analysis. In section 3, we discuss the light DM production from the inflaton decay. In section 4, we discuss several possibilities of the light DM produced by our mechanism, i.e., axion DM and right-handed neutrino DM. In section 5, we give an example of the inflaton model which gives $T_{R} \gtrsim m_{\phi}$. Section 6 is devoted to conclusions and discussion.

\section{Basic formalisms}

Throughout this paper we will use a Boltzmann equation, which governs the evolution of the distribution function of the DM particle, to describe the DM production from the inflaton decay. In this section, we introduce and solve the Boltzmann equation in expanding Universe to discuss the DM abundance produced by the decay of a scalar field $\phi$ (which is identified as the inflaton). We also discuss some relations between the Boltzmann equation and resonance parameter, which is introduced for a microscopic picture.

Throughout this paper, we assume that the potential of $\phi$ is well approximated by a parabolic one and that $\phi$ is spatially homogeneous. Then, for the timescale much shorter than the cosmic expansion, the motion of $\phi$ is given by

$$
\phi(t)=\bar{\phi} \cos m_{\phi} t
$$

\footnotetext{
${ }^{1}$ The parent scalar field does not have to be the inflaton; if it once dominates the universe, any scalar field may play the role. For the minimality of the scenario, in the this analysis, we assume that the parent scalar field is the inflaton.
} 
where $\bar{\phi}$ is the amplitude while $m_{\phi}$ is the mass of $\phi$. The number density of $\phi$ is given by

$$
n_{\phi}=\frac{1}{2} m_{\phi} \bar{\phi}^{2} .
$$

The Boltzmann equation of $\chi$ in the expanding Universe is given as

$$
\dot{f}_{\vec{k}}=H \vec{k} \frac{\partial f_{\vec{k}}}{\partial \vec{k}}+\dot{f}_{\vec{k}}^{(\text {coll })}
$$

where $H$ is the expansion rate of the universe:

$$
H=\frac{\dot{a}}{a},
$$

with $a$ being the scale factor, $f_{\vec{k}}$ is a distribution function of $\chi$ with three momentum $\vec{k}$, and $\dot{f}_{\vec{k}}^{(\text {coll) }}$ is the collision term (see, e.g. [42]). Hereafter, we concentrate on the case that the DM production is via the two-body decay of the inflaton, $\phi \rightarrow \chi \chi$. In such a case, ${ }^{2}$ the collision term is given by

$$
\dot{f}_{\vec{k}}^{(\text {coll })}=2 n_{\phi} \Gamma_{\phi \rightarrow \chi \chi}^{(0)}\left[\left(1 \pm f_{\vec{k}}\right)\left(1 \pm f_{-\vec{k}}\right)-f_{\vec{k}} f_{-\vec{k}}\right] \delta\left(|\vec{k}|-p_{\chi}\right)\left(\frac{p_{\chi}^{2}}{2 \pi^{2}}\right)^{-1},
$$

where, in the square bracket, $(1+f)$ and $(1-f)$ are for bosonic and fermionic DM, respectively, and $\Gamma_{\phi \rightarrow \chi \chi}^{(0)}$ is the decay rate. The superscript "(0)" indicates that $\Gamma_{\phi \rightarrow \chi \chi}^{(0)}$ is the perturbative decay rate at the vacuum. In addition, $p_{\chi}$ is the three-momentum of $\chi$ produced by the decay:

$$
p_{\chi} \equiv \frac{1}{2} m_{\phi} \sqrt{1-\frac{4 m_{\chi}^{2}}{m_{\phi}^{2}}} .
$$

The first and second terms in the square bracket in eq. (2.5) are understood as the contributions of the decay and the inverse decay (taking into account the stimulated emission or the Pauli blocking), respectively. ${ }^{3}$ Using the distribution function, the number density of $\chi$ is given by

$$
n_{\chi}(t)=g \int \frac{d^{3} k}{(2 \pi)^{3}} f_{\vec{k}}(t)
$$

with $g$ being the multiplicity. If $\chi$ is a real scalar and a singlet Weyl fermion, $g=1$ and 2 , respectively. When $g>1, \Gamma_{\phi \rightarrow \chi \chi}^{(0)}$ is defined as the decay rate to a single $\chi$ pair; it is not the decay rate to $g$ pairs of $\chi$.

\footnotetext{
${ }^{2}$ We consider the case that $n_{\phi}$ is so large that we can omit " 1 " in the Bose-enhancement factor for $\phi$ in the inverse decay process. It is also assumed that the change of $\bar{\phi}$ is so slow that $\bar{\phi}$ can be well approximated to be a constant in deriving collision term.

${ }^{3}$ Notice that, in eq. (2.5), the factor of 2 in the right-hand side reflects the fact that two $\chi$ 's are produced by the decay of single $\phi$. One can see that, for $f_{\vec{k}} \ll 1$,

$$
\dot{n}_{\chi}^{(\mathrm{coll})} \equiv g \int \frac{d^{3} k}{(2 \pi)^{3}} \dot{f}_{\vec{k}}^{(\mathrm{coll})} \simeq 2 g n_{\phi} \Gamma_{\phi \rightarrow \chi \chi}^{(0)} .
$$
}


Now, we estimate the DM abundance from the decay of $\phi$, taking into account the effects of the cosmic expansion. In the following, the distribution of $\chi$ is assumed to be isotropic so that the distribution function does not depend on the direction of $\vec{k}$ but depends only on $k \equiv|\vec{k}|$. Hereafter, the distribution function is denoted as $f_{k}$. In solving the Boltzmann equation in the expanding universe, it is convenient to introduce the comoving momentum:

$$
\hat{k}(k) \equiv \frac{a(t)}{a_{0}} k,
$$

where $a_{0}$ is the scale factor at some reference time. Let us define:

$$
\hat{f}_{\hat{k}}(t) \equiv f_{k}(t)
$$

Then, $\hat{f}_{\hat{k}}$ satisfies the following differential equation:

$$
\dot{\hat{f}}_{\hat{k}}=\frac{2 n_{\phi} \Gamma_{\phi \rightarrow \chi \chi}^{(0)}}{H p_{\chi}}\left(1 \pm 2 \hat{f}_{\hat{k}}\right) \delta\left(t-t_{\hat{k}}\right)\left(\frac{p_{\chi}^{2}}{2 \pi^{2}}\right)^{-1} .
$$

Here, $t_{\hat{k}}$ is the cosmic time when $k$ becomes equal to $p_{\chi}$ for a given $\hat{k}$, i.e., it is given by

$$
a\left(t_{\hat{k}}\right)=a_{0} \frac{p_{\chi}}{\hat{k}}
$$

Solving eq. (2.10) with $f_{k}(t \rightarrow 0)=0$, we obtain ${ }^{4}$

$$
f_{k}(t \rightarrow \infty)= \pm \frac{1}{2}\left(e^{ \pm 2 \bar{f}\left(t_{k}\right)}-1\right) \theta\left(p_{\chi}-k\right),
$$

with

$$
\left.\bar{f}\left(t_{k}\right) \equiv \frac{4 \pi^{2} \Gamma_{\phi \rightarrow \chi \chi}^{(0)} n_{\phi}}{H p_{\chi}^{3}}\right|_{t=t_{\hat{k}}} .
$$

For the case of bosonic DM, the production of the DM is exponentially enhanced when $\bar{f} \gtrsim 1$ due to the stimulated emission. This is like the light amplification by stimulated emission of radiation, LASER; we call the DM amplification by stimulated emotion of radiation (i.e., relativistic DM) as DASER.

Before closing this section, we comment on the Boltzmann equation from the point of view of parametric resonance. To this end, we assume the interacting Lagrangian

$$
\mathcal{L}_{\text {int }}=-A \phi \chi^{2}
$$

\footnotetext{
${ }^{4}$ When $\bar{f} \gg 1$, in fact, a quantum effect may become important, which reduces the exponent by a factor of $1 / 2$. For more discussion about the particle production in the framework of the quantum field theory, as well as the relation between the parametric resonance and the Bose-enhancement factor in the Boltzmann equation, see [41]. Although the quantum effect is important for a precise calculation of the DM abundance, it does not affect our discussion in the following sections.
} 
for the interaction of $\phi$ with a real scalar field $\chi$. Then the decay rate is obtained as

$$
\Gamma_{\phi \rightarrow \chi \chi}^{(0)}=\frac{A^{2}}{8 \pi m_{\phi}} \frac{2 p_{\chi}}{m_{\phi}} .
$$

Further, for simplicity, we neglect the mass of $\chi$, and thus $m_{\phi} \simeq 2 p_{\chi}$.

The production of $\chi$ in this system has been also studied in the context of parametric resonance [43-46]. In the particle production through the parametric resonance, the particles in resonance bands are effectively produced. The widths of the bands are typically of $O\left(q m_{\phi}\right)$, where

$$
q \equiv \frac{4 A \bar{\phi}}{m_{\phi}^{2}}
$$

We study the case that the band widths are narrow, i.e.,

$$
q \ll 1
$$

Then, the DM particles are produced almost "on-resonance," i.e., the momentum of the DM produced from the inflaton oscillation is $|\vec{k}| \simeq p_{\chi}$. Otherwise, the DM acquires an oscillating mass with amplitude larger than $m_{\phi}$ and the aforementioned decay process is sometimes forbidden.

A given comoving momentum stays in the resonance band for the timescale of $O(q / H)$. Thus, for the validity of our argument, the change of the inflaton amplitude during such a timescale should be negligible, which requires

$$
\frac{\dot{\bar{\phi}}}{\bar{\phi}} \ll q^{-1} H .
$$

We also note here that the exponential growth shown in eq. (2.12) is consistent with the behavior observed in the analysis of parametric resonance in the narrow resonance regime, $q \ll 1$ [44]. In fact, there exist processes which are higher order in $\mathcal{L}_{\text {int }}$. So far, we have considered the effects of $O\left(A^{2}\right)$; in the perturbative picture, they take account of the decay and inverse decay processes $\phi \leftrightarrow \chi \chi$. Effects which are higher order in $A$ are due to scattering processes, in which a number of $\phi$ 's annihilate into $\chi$ 's. Such effects are suppressed when the amplitude of $\phi$ is small enough; the higher order terms are suppressed by powers of $q$ and are negligible when $q \ll 1$ [47].

\section{Light DM from inflaton decay}

\subsection{Cold DM from inflaton decay}

Now we consider the DM production from the inflaton decay, $\phi \rightarrow \chi \chi$. The DM is assumed to have negligible interaction rate with itself and with Standard Model (SM) particles. ${ }^{5}$

\footnotetext{
${ }^{5}$ This condition may be favored because the DM should be cold and abundant throughout the thermal history, e.g. they should not be heated by the ambient SM plasma or by itself [48].
} 
The inflaton, on the other hand, not only couple to the DM but also couples to some SM particles. The latter coupling leads to the reheating of the Universe.

The energy density of the universe is once dominated by that of the inflaton oscillation after inflation/preheating. Here, we assume that the inflaton potential is well approximated by the quadratic one in such a period (cf. [49-55]). In such a case, the inflaton energy density $\rho_{\phi}$ scales as $a^{-3}$ during the period of inflaton oscillation. We denote the cosmic time at the beginning of the inflaton oscillation as $t_{i}$, and that at the completion of the reheating as $t=t_{R}$. (Thus, at $t=t_{R}$, energy density of the inflaton is converted to that of radiation and the radiation-dominated universe starts.) In the following, for simplicity, we adopt the sudden-decay approximation, i.e., the inflaton is approximated to decay at $t=t_{R}$ for the estimation of the reheating temperature.

During the oscillating period, the inflaton mass should be larger than the expansion rate of the universe $H$. Then, based on the inequality $m_{\phi} \gg H\left(t_{R}\right) \sim \sqrt{g_{\star} \pi^{2} / 90} T_{R}^{2} / M_{\mathrm{pl}}$ (with $g_{\star}$ being the effective number of relativistic degrees of freedom), we obtain a constraint on the reheating temperature:

$$
T_{R} \lesssim T_{R}^{(\max )} \equiv\left(\frac{g_{\star} \pi^{2}}{90}\right)^{-1 / 4} \frac{M_{\mathrm{pl}}}{r} \simeq 10^{12} \mathrm{GeV} \times\left(\frac{r}{10^{6}}\right)^{-1},
$$

where

$$
r \equiv \frac{T_{R}}{m_{\phi}}
$$

Notice that $T_{R}^{(\max )}$ is defined as the maximal possible reheating temperature for a fixed value of $r$.

We introduce the parameter $n_{\phi}^{(0)}\left(t_{R}\right)$ such that the energy density of the inflaton for $t_{i} \ll t \ll t_{R}$ is well approximated as

$$
\rho_{\phi}(t) \simeq m_{\phi} n_{\phi}^{(0)}\left(t_{R}\right)\left(\frac{a\left(t_{R}\right)}{a(t)}\right)^{3} .
$$

Then, we define the effective number of DM produced by one inflaton as

$$
B \equiv \frac{n_{\chi}\left(t_{R}\right)}{n_{\phi}^{(0)}\left(t_{R}\right)}
$$

Here, $n_{\chi}\left(t_{R}\right)=a\left(t_{R}\right)^{-3} \int_{t_{\text {inf }}}^{t_{R}} d t n_{\phi}(t) a(t)^{3} \Gamma_{\phi \rightarrow \chi}(t)$, where $\Gamma_{\phi \rightarrow \chi}(t)$ is the number density transferring rate, and $t_{\text {inf }}$ is the time at the end of inflation. This formula includes the production during the preheating epoch. Using $B$, the number density of DM for $t \gtrsim t_{R}$ is given by

$$
n_{\chi}(t)=B n_{\phi}^{(0)}\left(t_{R}\right)\left(\frac{a\left(t_{R}\right)}{a(t)}\right)^{3}
$$

Notice that, as we will discuss in the following, the DM density may be dominated by those produced at the beginning of the oscillating period. In such a case, $B$ can be much larger 
than the branching fraction $\operatorname{Br}(\phi \rightarrow \chi \chi)$. Using the fact that the entropy density $s$ scales as $a^{-3}$ after the reheating, we can estimate the density parameter of DM as

$$
\Omega_{\chi} \sim \frac{m_{\chi} n_{\chi}\left(t_{R}\right)}{s\left(t_{R}\right)}\left(\frac{\rho_{\text {crit }}}{s_{0}}\right)^{-1} \sim \frac{3}{4} m_{\chi} r B \frac{s_{0}}{\rho_{\text {crit }}}
$$

where $s_{0}$ is the present entropy density and $\rho_{\text {crit }}$ is the critical density. Numerically, we obtain

$$
m_{\chi} \sim 0.26 \mathrm{eV} \times \frac{1}{r B}\left(\frac{\Omega_{\chi} h^{2}}{0.12}\right),
$$

with $h$ being the Hubble constant in units of $100 \mathrm{~km} / \mathrm{sec} / \mathrm{Mpc}$.

Next, let us consider the coldness of DM. In the following, for simplicity, we assume that the reheating is instantaneous, i.e., $t_{R} \sim t_{i}$. (For the case of $t_{R} \gg t_{i}, \chi$ can be also produced at earlier epoch and is colder due to an extra red-shift.) In addition, we consider the case that $m_{\chi} \ll m_{\phi}$.

At the time of the inflaton decay (i.e., $T \sim T_{R}$, with $T$ being the cosmic temperature), the produced DMs are relativistic and their momenta are typically

$$
P_{\chi}\left(T_{R}\right) \sim \frac{1}{2} m_{\phi}
$$

where we have assumed that the DM production is dominated at $t \sim t_{R}$. Notice that, if the DMs are mostly produced at $t \ll t_{R}, P_{\chi}\left(T_{R}\right)$ is more suppressed and DMs become colder. With the expansion of the universe, the momentum is red-shifted as $P_{\chi}(T)=$ $\frac{g_{\star, s}(T)^{1 / 3} T}{g_{\star, s}\left(T_{R}\right)^{1 / 3} T_{R}} P_{\chi}\left(T_{R}\right)$ (with $g_{\star, s}$ being the effective number of relativistic degrees of for the entropy density), and hence the velocity of the DM is estimated as

$$
v(T) \sim \min \left(\frac{g_{\star, s}(T)^{1 / 3} T}{2 g_{\star, s}\left(T_{R}\right)^{1 / 3} m_{\chi}} r^{-1}, 1\right)
$$

If the DM mass is too small, the DM velocity becomes too large to be consistent with the Lyman- $\alpha$ bound. Here, we adopt the analysis on a warm DM of sterile neutrino $[5,6]$ to estimate the lower bound on the DM mass. Regarding the constraint on the root mean square velocity of the warm sterile neutrino $\mathrm{DM} \sqrt{\left\langle v_{\text {warm }}(T)^{2}\right\rangle}$ as that on $v(T)$ of our model, we estimate the lower bound of the DM mass. (See a similar approach to the FIMP dark matter [7].) Then, we obtain

$$
m_{\chi} \gtrsim 0.9 \mathrm{keVr}^{-1}\left(\frac{107.75}{g_{\star, s}\left(T_{R}\right)}\right)^{1 / 3},
$$

where, to be conservative, we adopt the lower bound on the sterile neutrino mass of $\sim$ $2 \mathrm{keV}[5]$. In the analysis in section 3.2, it is not always the case that the DM production is dominated when $t \sim t_{R}$, and hence we will calculate the root mean square velocity using the actual DM spectrum to derive the bound. 


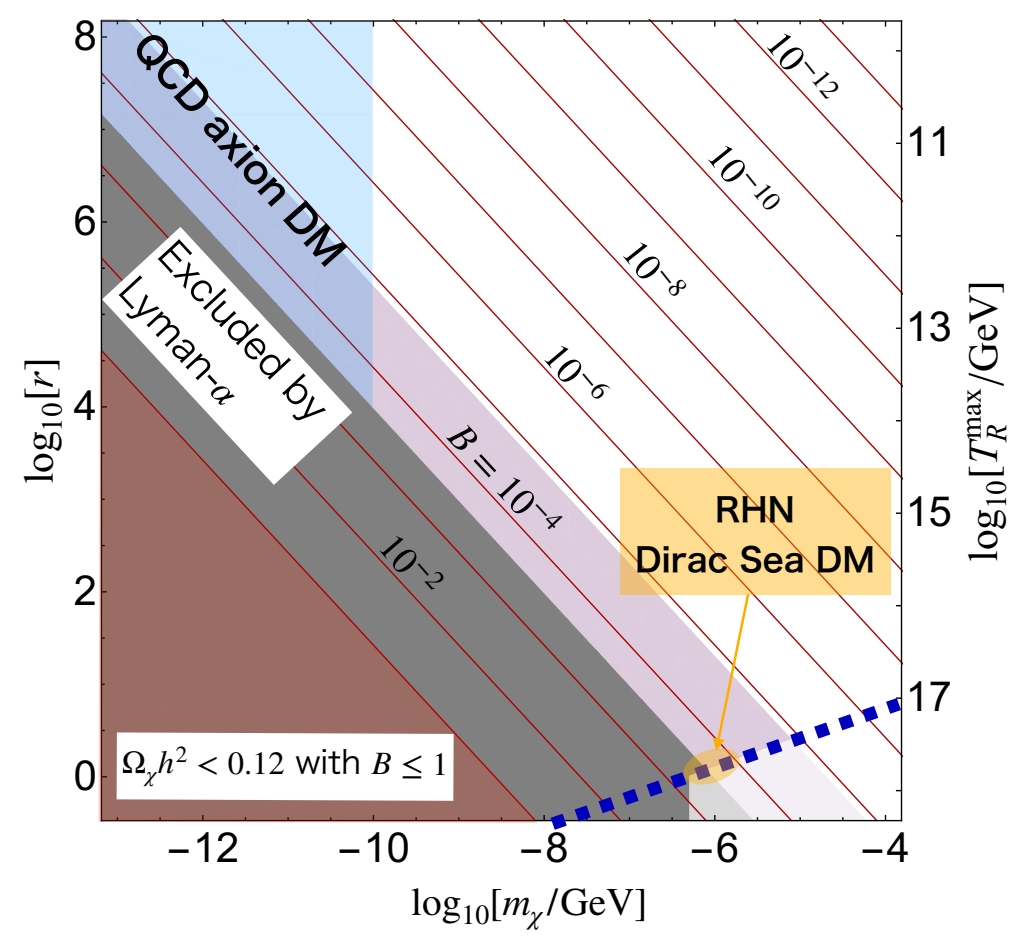

Figure 1. The contour of constant $B$ to realize the present DM density on $m_{\chi}$ vs. $r$ plane (see eq. (3.7)), taking $\Omega_{\chi} h^{2}=0.12$. The maximal possible reheating temperature as a function of $r$ is shown on the right axis. In the gray region, the DM may be too hot to be consistent with Lyman- $\alpha$ forest data by assuming that the DM momenta at $t=t_{R}$ is typically $m_{\phi} / 2$. The purple region may be tested in the future. For the case of the Fermionic DM, only the region below the blue-dotted line is relevant because of the Pauli-blocking (see section 3.2). The typical parameter range of QCD axion DM and right-handed neutrino DM are shown in light blue and orange (see section 4).

When the DM is fermionic, there is a so-called Tremaine-Gunn (TG) bound [56, 57]:

$$
m_{\chi} \gtrsim 0.5 \mathrm{keV}\left(\frac{2}{g}\right)^{1 / 4}
$$

which is derived from the upper-bound on the phase space density in dwarf spheroidal galaxies. For $\chi$ forming a Dirac sea, this bound is comparable to the recast Lyman- $\alpha$ bound [58].

From eqs. (3.7) and (3.10), we get the key observation that, even if the DM has a small mass, the coldness of the DM can be realized with avoiding the Lyman- $\alpha$ bound taking $r \gtrsim 1$. We emphasize that $r \gtrsim 1$ is easily realized if the inflaton-SM coupling is large enough. In particular, $r \gg 1$ can be obtained when a dissipation effect, which is a scattering process between the inflaton condensate and the SM plasma, is important to complete the reheating [23-29]. A general prediction in this dissipation regime is that the inflaton is thermalized at the end of the reheating epoch. The dissipation effect is rather generic and our conclusion below does not depend on the detail of the model. However, we provide a concrete model in section 4 .

The discussion so far is summarized in figure 1 on $m_{\chi}$ vs. $r$ plane. In figure 1 , the contours of constant $B$ which satisfy the DM constraint given in eq. (3.7) are shown. On the 
right axis, the aforementioned model-independent maximal reheating temperature, $T_{R}^{(\max )}$, is shown. The recast Lyman- $\alpha$ bound (3.10) is shown as gray-shaded region, while the current DM abundance cannot be realized in the red-shaded region if $B \leq 1$. One can see that the recast Lyman- $\alpha$ bound fully excludes the parameter region with $B \geq 1$ and hence we do not consider such a value of $B$. The purple region may be tested by the future $21 \mathrm{~cm}$ line observation [59]; the region corresponds to the warm sterile neutrino DM with $m_{N} \lesssim 20 \mathrm{keV}$. Thus, if $r$ is large enough, the DM produced by the inflaton decay can be cold and abundant enough to be consistent with observations. We note here that all the region in the figure is applicable to a bosonic DM; for bosonic DM, the effect of the Bose enhancement becomes significant in the region above the blue dotted line. For the case of a fermionic DM, on the contrary, only the region below the blue dotted line is relevant, as we will discuss in the following.

\subsection{DM production in a narrow resonance regime}

Now let us estimate the parameter $B$. Given an inflation model and inflaton coupling to $\chi$, we can estimate $B$.

In general, $B$ can be expressed as

$$
B=B^{(\mathrm{preh})}+B^{(\mathrm{reh})},
$$

where $B^{(\text {preh })}$ and $B^{(\text {reh })}$ are contributions during the preheating (i.e., $t \lesssim t_{i}$ ) and during the inflaton oscillation (i.e., $t_{i} \lesssim t \lesssim t_{R}$ ), respectively. $B^{(\mathrm{preh})}$ contributes to the IR mode of the DM distribution function $f_{k}$. The preheating epoch is highly model dependent. For instance, in the inflation models such as small-field inflation and hybrid inflation, tachyonic preheating generally takes place and the inflaton zero mode settles into the quadratic regime within $O(1)$ oscillation [60-62]. ${ }^{6}$ Hereafter we do not consider $B^{(\mathrm{preh})}$, and concentrate on the effects after the inflaton oscillation becomes effective. Thus, we mostly consider the case of $B \sim B^{(\mathrm{reh})}$.

Now we estimate the DM abundance produced during the inflaton oscillation. The production in this epoch is somewhat model-independent if the system is in narrow resonance regime, i.e., $q \ll 1$. Using the results given in section 2 , the number density of the DM at time of the reheating is estimated as

$$
n_{\chi}^{(\mathrm{reh})}\left(t_{R}\right) \sim \pm \frac{1}{2} g \int_{k_{\mathrm{IR}}}^{p_{\chi}} \frac{d^{3} k}{(2 \pi)^{3}}\left(e^{ \pm 2 \bar{f}\left(t_{k}\right)}-1\right),
$$

where $k_{\mathrm{IR}}$ represents the momentum of DM produced at $t \sim t_{i}$, i.e., $k_{\mathrm{IR}} \sim \frac{a\left(t_{i}\right)}{a\left(t_{R}\right)} p_{\chi}$. If $\bar{f} \ll 1$ during the whole reheating epoch, we obtain $B^{(\mathrm{reh})} \sim 2 t_{R} \Gamma_{\phi \rightarrow \chi \chi}^{(0)}$. This result does not depend whether the DM is a fermion or a boson.

\footnotetext{
${ }^{6}$ In this case, there are also produced spatial inhomogeneous modes, which have comparable energy to the inflaton condensate and are non-relativistic [60-62]. The decays of the inhomogeneous modes may slightly modify the spectra, $f_{k}$, but we expect our estimation does not change much. Also, the field value of $\phi$ just after inflation is far away from the minimum. If we use the interaction given in eq. (2.14), the DM may either receives a heavy positive or negative mass squares depending on the sign of $A$. The latter case may generate the DM via another tachyonic preheating. This should correspond to a hybrid inflation.
} 


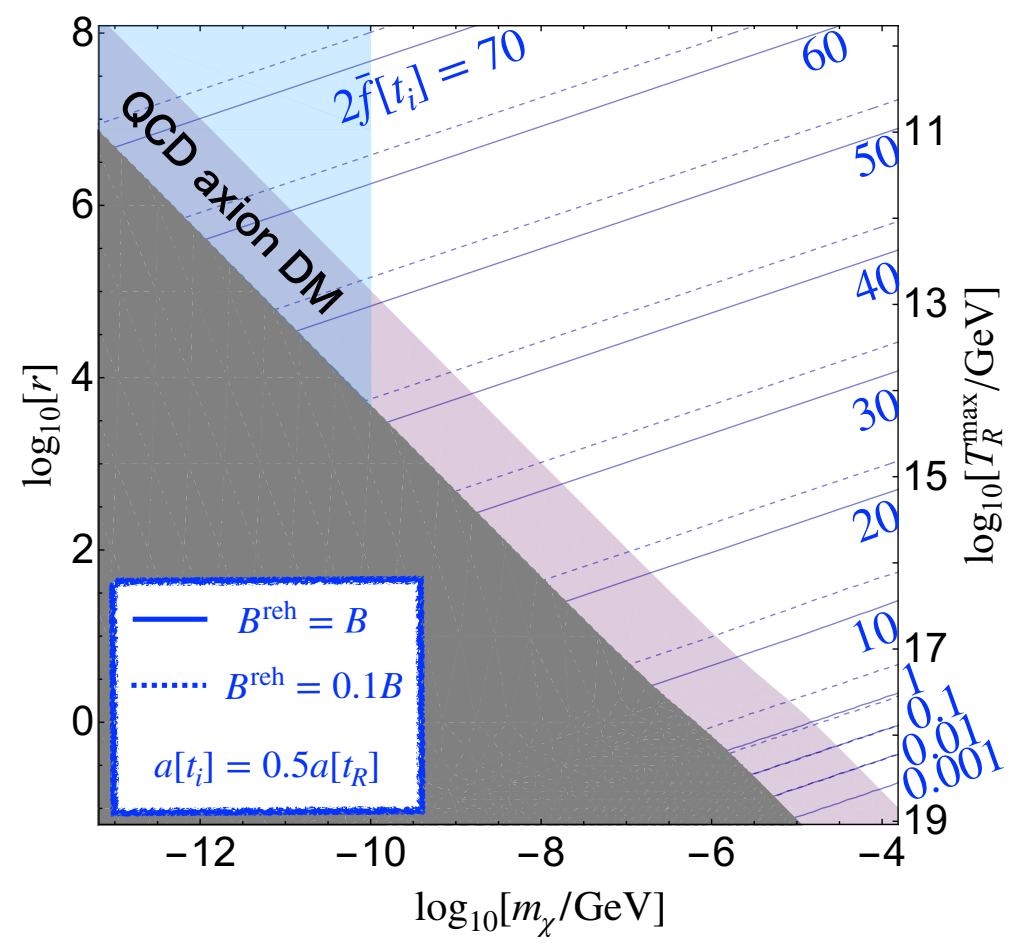

Figure 2. The contour of $2 \bar{f}\left(t_{i}\right)$ of a bosonic DM in $m_{\chi}-r$ plane by requiring $\Omega_{\chi} h^{2}=0.12$ with $B^{(\mathrm{reh})}=B$ (blue solid) and $B^{(\mathrm{reh})}=0.1 B$ (blue dotted). We assume $a\left(t_{i}\right)=0.5 a\left(t_{R}\right)$. See figure 1 for the meanings of the color-shaded regions.

The case with $\bar{f} \gtrsim 1$ is interesting. In this case, the exponential nature in eq. (3.13) becomes important and $B$ depends on the statistics of $\chi$. When $\chi$ is bosonic, the integral is dominated at $k \sim k_{\mathrm{IR}}$; in other words, the production of the DM becomes most efficient at the beginning of the reheating at which the effect of the Bose enhancement becomes the largest because $\bar{f} \propto n_{\phi} / H \propto a^{-3 / 2}$. Due to the exponential enhancement, $B^{(\mathrm{reh})} \gtrsim$ $2 t_{R} \Gamma_{\phi \rightarrow \chi \chi}^{(0)}$. Thus, the DM production during the reheating era is enhanced via the DASER mechanism. Since $e^{2 \bar{f}}$ changes rapidly as a function of time, $\bar{f}$ does not have to be much larger than unity to obtain the value of $B$ given in figure 1 . In figure 2 , we show the contours of constant $2 \bar{f}\left(t_{i}\right)$ to satisfy eq. (3.7), assuming $a\left(t_{i}\right)=0.5 a\left(t_{R}\right)$. On the blue solid and dashed contours, $B^{(\mathrm{reh})}=B$ and $B^{(\mathrm{reh})}=0.1 B$ are assumed, respectively. In the latter case, the contribution from the preheating epoch may be dominant. Notice that, for the case of $a\left(t_{i}\right)=0.5 a\left(t_{R}\right)$, the red shift during the inflaton oscillation period is short and $n_{\chi}\left(t_{R}\right) \sim O\left(g p_{\chi}^{3} e^{2 \bar{f}\left(t_{R}\right)}\right)$. Then, we can find that the value of $e^{2 \bar{f}\left(t_{R}\right)}$ giving rise to the relevant DM density scales as $\propto\left(r^{3} / m_{\chi}\right)$. Numerically, for the case of $\bar{f}\left(t_{i}\right) \gtrsim 1$, we find that figure 2 gives:

$$
e^{2 \bar{f}\left(t_{i}\right)} \sim 3 \times 10^{23}\left(3 \times 10^{22}\right) \times\left(\frac{r}{10^{6}}\right)^{3}\left(\frac{m_{\chi}}{10^{-8} \mathrm{GeV}}\right)^{-1},
$$

to make $\Omega_{\chi}$ to be consistent with the DM density parameter with $B^{(\mathrm{reh})}=B\left(B^{(\mathrm{reh})}=0.1 B\right)$. This fit is relevant for the parameter region with $r \gtrsim 1$. We find that our prediction of $\bar{f}$ 
does not change much even if the contribution from the preheating epoch is sizable. ${ }^{7} \mathrm{We}$ also use the distribution function given in eq. (3.13) to numerically estimate the averaged velocity, $\sqrt{\left\langle v^{2}\right\rangle}$, with $B=B^{(\text {reh })}$ to recast the Lyman- $\alpha$ constraint and the future reach of $21 \mathrm{~cm}$ line observation, which are shown in the figure by the gray and pink regions, respectively.

For the case that the DM is fermionic, on the other hand, the Pauli-blocking effect should be taken into account if $\bar{f} \gtrsim 1$. Thus, the number density of the fermionic DM has an upper-bound:

$$
n_{\text {fermion } \chi}^{(\max }\left(t_{R}\right)=g \int_{k \leq p_{\chi}} \frac{d^{3} k}{(2 \pi)^{3}}=\frac{g m_{\phi}^{3}}{96 \pi^{2}} .
$$

This leads to

$$
B \leq \frac{m_{\phi} n_{\text {fermion } \chi}^{(\max )}\left(t_{R}\right)}{\rho_{\phi}\left(t_{R}\right)} \sim 6 \times 10^{-4} r^{-4} g\left(\frac{11}{g_{\star}}\right) \quad: \quad \text { fermionic DM. }
$$

In figure 1, the above constraint is shown by the blue dotted line. In addition, combining eq. (3.16) with eq. (3.7), we obtain the DM mass to realize the Dirac sea DM as

$$
m_{\chi} \sim 0.4 \mathrm{keV} r^{3}\left(\frac{2}{g}\right)\left(\frac{g_{\star, s}}{11}\right) \quad: \quad \text { Dirac sea DM. }
$$

So far, we have not considered back-reaction from plasma effect in the $\chi$ production because the interaction of $\chi$ with the SM particles or $\chi$ are assumed to be negligible. Also, the plasma effect induced by interaction between $\chi$ and thermalized $\phi$ is suppressed due to the small coupling in the narrow resonance regime, e.g. with the Lagrangian (2.14), the thermal correction in the dispersion relation of $\chi$ should be $\delta m_{\chi}^{2} \sim O\left(A^{2}\right) \ll q^{2} m_{\phi}^{2}$.

\section{DM models}

In this section, we consider two DM models in which our mechanism plays an important role, i.e., QCD axion and right-handed neutrino (RHN).

\subsection{DASER production of isocurvature free axion DM}

We first consider axion (-like) particle $a$. (Thus, in this subsection, the DM particle is denoted as a.) The well-known production mechanism of the axion DM is the misalignment mechanism [8-10]. That is, when the (effective) axion mass becomes comparable to the expansion rate of the universe, axion starts to oscillate around the potential minimum. The coherent oscillation of axion becomes the cold DM. However, if the PQ breaking scale is small, the initial amplitude is suppressed and the resulting abundance, $\Omega_{a}^{(\mathrm{mis})}$, is

\footnotetext{
${ }^{7}$ If the inflaton condensate is significantly fragmented during the preheating (see footnote 6), we may need $\bar{f}\left(t_{i}\right)$ larger than the value given in eq. (3.14) because the exponential enhancement may not as strong as inflaton decay. However, a certain level of the exponential growth is needed since the DM number within the sphere of radius $m_{\phi} / 2$ is over occupied to explain the DM abundance. Thus we expect again that $2 \bar{f}$ does not have to be much larger to realize the relevant DM density.
} 
suppressed. If the PQ breaking happens before the inflation, the quantum fluctuation of the axion may also contribute to the power spectrum of isocurvature perturbation. Our mechanism is complementary to the misalignment mechanism and it works even when $\Omega_{a}^{(\mathrm{mis})}$ is smaller than the density parameter of the DM.

In the following, we will show that the DASER production during reheating may provide an alternative mechanism to produce cold axion DM. For concreteness, we adopt the following higher-dimensional operator for the inflaton coupling to axion:

$$
\mathcal{L}_{\text {int }}=-\frac{\phi}{\Lambda_{a}} \partial_{\mu} a \partial^{\mu} a
$$

where $\Lambda_{a}$ is a constant. The higher dimensional operator given above can originate from the coupling $\frac{2 \phi}{\Lambda_{a}} \partial_{\mu} \Phi_{\mathrm{PQ}}^{*} \partial^{\mu} \Phi_{\mathrm{PQ}}$, where $\Phi_{\mathrm{PQ}}$ is the PQ field responsible for the breaking of the $\mathrm{U}(1)_{\mathrm{PQ}}$ symmetry. Alternatively, the $\phi \rightarrow a a$ decays can be possible through the mixing between $\phi$ and the PQ Higgs boson via the renormalizable coupling $\phi\left|\Phi_{\mathrm{PQ}}\right|^{2}$. In both cases, the $\phi-a-a$ coupling is obtained by integrating out the heavy PQ Higgs boson which is assumed to be heaver than $\phi$. Even though the interaction given in eq. (4.1) has a different form from that in eq. (2.14), the discussion on resonance parameters in previous sections are applicable with replacing $A \rightarrow \frac{m_{\phi}^{2}}{2 \Lambda_{a}}$. Then, the decay rate of $\phi$ into the axion pair is given by

$$
\Gamma_{\phi \rightarrow a a}^{(0)}=\frac{1}{32 \pi} \frac{m_{\phi}^{3}}{\Lambda_{a}^{2}}
$$

Although our mechanism works for a large class of axion models, we focus on a QCD axion DM. The QCD axion should be in the following mass window [8-10, 63-65]:

$$
10^{-6} \mathrm{eV} \lesssim m_{a} \lesssim 0.1 \mathrm{eV}
$$

where the lower limit is from the mass density of the coherent oscillation of the axion assuming that the initial misalignment angle $\theta_{i}$ is of $O(1),{ }^{8}$ while the upper limit is from the duration of neutrino burst in SN1987A. By using misalignment mechanism to obtain the axion DM with $m_{a} \gg 10^{-6} \mathrm{eV}, \theta_{i}$ should be fine-tuned so that the axion initially stays (almost) at the top of the potential to enhance the abundance due to an anharmonic effect $[66,67]$. In such a case, the power spectrum of the axion isocurvature perturbation is significantly enhanced $[68,69]$ and the isocurvature problem, as well as the domain-wall problem [70], may be serious. In particular, for $m_{a} \gtrsim 10^{-3} \mathrm{eV}$, to evade the isocurvature bound, the energy density during inflation is smaller than $\mathrm{MeV}^{4}$, which is not consistent with the big-bang nucleosynthesis (BBN).

The DASER production mechanism gives a new possibility to realize axion DM with $m_{a} \gg 10^{-6} \mathrm{eV}$. To realize the value of $\bar{f}$ in eq. (3.14), the model parameters are related as

$$
2 \bar{f} \sim 50 \times\left(\frac{1.7 \times 10^{14} \mathrm{GeV}}{\Lambda_{a}}\right)^{2}\left(\frac{r}{10^{5}}\right)\left(\frac{T_{R}}{10^{5} \mathrm{GeV}}\right),
$$

\footnotetext{
${ }^{8}$ Here we have assumed $H_{\text {inf }} \gg 1 \mathrm{GeV}$. If $H_{\text {inf }} \ll 1 \mathrm{GeV}$ and inflation lasts long enough, $\left|\theta_{i}\right| \ll 1$ follows from the equilibrium distribution during inflation, and this bound disappears [13, 14].
} 
where, in this calculation, an (almost) instantaneous reheating is assumed: $\rho_{\phi}\left(t_{i}\right) \sim \frac{g_{\star} \pi^{2}}{30} T_{R}^{4}$. If $t_{R} \ll t_{i}$, the required $\Lambda_{a}$ becomes larger. Therefore even if the inflaton coupling is weak, $B$ can be large enough to have a dominant component of the axion DM from the DASER production mechanism.

As we discuss in section 2, our analysis is valid when the timescale of the dissipation is longer than $\sim\left(q m_{\phi}\right)^{-1} \sim \Lambda_{a} /\left(\bar{\phi} m_{\phi}\right)$ (see eq. (2.18) with $H \sim q^{2} m_{\phi}$ for DASER production). In the case of the instantaneous reheating, $t_{R} \sim t_{i} \sim 1 / \Gamma_{\text {dis }}$, with $\Gamma_{\text {dis }} \gtrsim H\left(t_{R}\right)$, where $\Gamma_{\text {dis }}$ is the dissipation rate for the reheating. Thus, we require $\Gamma_{\text {dis }} \lesssim \bar{\phi} m_{\phi} / \Lambda_{a}$.

The DASER axion from the inflaton decay does not have an isocurvature problem. This is because the axion density fluctuation follows that of the inflaton. In the model of section 5 , the axion DM in the range of $10^{-4} \mathrm{eV} \lesssim m_{a} \lesssim 10^{-1} \mathrm{eV}$ can be generated. Such axion can give signal in DM haloscopes [71-75], IAXO experiment [76-78], and ARIADNE experiment $[79,80]$.

The DASER axion may be thermalized due to the scattering with the ambient plasma of the SM particles. Assuming that the axion only couples to the gluon (except for the inflaton) below the PQ scale, we obtain the dissipation rate which is suppressed due to the nature of derivative couplings (by neglecting a QCD sphaleron contribution [81]): $\Gamma_{\text {diss }}^{\text {axion }} \sim$ $\frac{\alpha_{s}^{2} T^{3}}{32 \pi^{2} f_{a}^{2}} \frac{P_{a}^{2}}{g_{s}^{4} T^{2}}$, where $P_{a}$ is the typical momentum of the axion at SM temperature, $g_{s}$ is the QCD coupling constant, and $f_{a}$ is the axion decay constant [29]. One finds that, for $T / P_{a} \sim 2 r \gg 1$, the rate is much smaller than the expansion rate $H$ if

$$
\frac{T}{f_{a}} \lesssim 0.1 \times\left(\frac{f_{a}}{10^{9} \mathrm{GeV}}\right)\left(\frac{r}{100}\right)^{2}
$$

In the parameter region with $T_{R} \ll f_{a}$ and $r \gg 100$, the DASER axion is not thermalized. ${ }^{9}$

The DASER mechanism is generic for bosonic particles which is weakly coupled to inflaton and SM particles; the candidates include hidden photon and axion-like particle DM. For instance, one can also use the DASER mechanism to produce the hidden photon or axion DM to explain the XENON1T excess [4, 82-88]. Conversely, such a DASER production process may overproduce stable (or long-lived) bosons which may result in cosmological problems with dark matters or dark radiations.

\subsection{Dirac sea DM of right handed neutrino}

RHNs are well-motivated particle to give masses to active neutrinos via the seesaw mechanism [89-93]. Denoting the left- and right-handed neutrinos as $\nu$ and $N$, respectively, the Lagrangian in the seesaw scenario contains the following terms:

$$
\mathcal{L} \supset-\frac{M_{N}}{2} \bar{N}^{c} N-y_{\nu} v \bar{N} \nu+\text { h.c. },
$$

where $v$ is the expectation value of the Higgs field. Here, for simplicity, we assume that only a single flavor of the RHN is important for our discussion. Then, $\nu$ can be regarded

\footnotetext{
${ }^{9}$ If $T_{R} \gtrsim f_{a}$, we should consider the effects of heavy particles like the PQ scalars and PQ fermions. We do not consider such a case.
} 
as a superposition of electron neutrino $\nu_{e}$, muon neutrino $\nu_{\mu}$, and tau neutrino $\nu_{\tau}$ :

$$
\nu=\epsilon_{e} \nu_{e}+\epsilon_{\mu} \nu_{\mu}+\epsilon_{\tau} \nu_{\tau}
$$

where $\epsilon_{i}$ 's, which are assumed to be real, denote coefficients satisfying $\sum_{i=e, \mu, \tau} \epsilon_{i}^{2}=1$. In the following, we focus on the case of $M_{N} \gg y_{\nu} v$. Then, by integrating out $N$, we obtain an active neutrino mass term:

$$
\mathcal{L}_{\text {eff }} \supset \frac{\left(y_{\nu} v\right)^{2}}{2 M_{N}} \bar{\nu}^{c} \nu \equiv \frac{m_{\nu}}{2} \bar{\nu}^{c} \nu .
$$

In this case, the mixing angle between the left- and right-handed neutrinos is given by

$$
\theta \equiv \frac{y_{\nu} v}{M_{N}} .
$$

From the data of neutrino-oscillation experiments, we can estimate [94]

$$
m_{\nu}=\theta^{2} M_{N} \sim(1-100) \mathrm{meV} .
$$

The RHN (which may be also called as "sterile neutrino") $N$ is a well-studied DM candidate [95, 96] (see also [97]). If $N$ carries the momenta comparable to the temperature of the active neutrinos, the Lyman- $\alpha$ constraint forces the right-handed neutrino mass $M_{N}$ to be heavier than $2-5 \mathrm{keV}$ as a warm DM $[5,6]$ (than $5-20 \mathrm{keV}$ as a FIMP DM [7]). For the RHN mass of our interest, the RHN decays as $N \rightarrow \gamma \nu$ with non-vanishing mixing angle $\theta$, and hence the mixing angle $\theta$ is required to be highly suppressed to evade the bound from $X$-ray observations [97]. It imposes a stringent constraint on the scenario of the RHN DM [97]. However, in our scenario, the RHN DM with sub keV mass may be produced as Dirac sea and can be the dominant DM component without conflicting the $X$-ray constraint as we see below.

Dirac sea of the RHN can be produced from the inflaton decay. The inflaton can have a coupling of the following form:

$$
\mathcal{L} \supset y_{\phi} \phi \bar{N}^{c} N
$$

where $y_{\phi}$ is a dimensionless Yukawa coupling constant. The decay rate is

$$
\Gamma_{\phi \rightarrow N N}^{(0)}=\frac{y_{\phi}^{2}}{4 \pi} m_{\phi}
$$

If $y_{\phi}$ is large enough, soon the decay process is Pauli-blocked. Then the decay products form the Dirac sea. Consequently the DM abundance can be explained with $r \sim 1-10$ for $m_{N}=0.1-100 \mathrm{keV}$ (see the blue dashed line of figure 1).

In figure 3, we show various constraints on the Dirac sea DM of the RHN on $M_{N}$ vs. $\theta^{2}$ plane. In the orange band, the see-saw relation given in eq. (4.10) is possible. The $X$-ray and TG bounds are taken from [97]. The sensitivities of the ECHo and KATRIN experiment are applicable to the case that the RHN dominantly mixes with the electron neutrino (i.e, $\epsilon_{e} \sim 1$ ); if $0.5 \mathrm{keV} \lesssim M_{N} \lesssim 2 \mathrm{keV}$, the existence of the RHN can affect the 


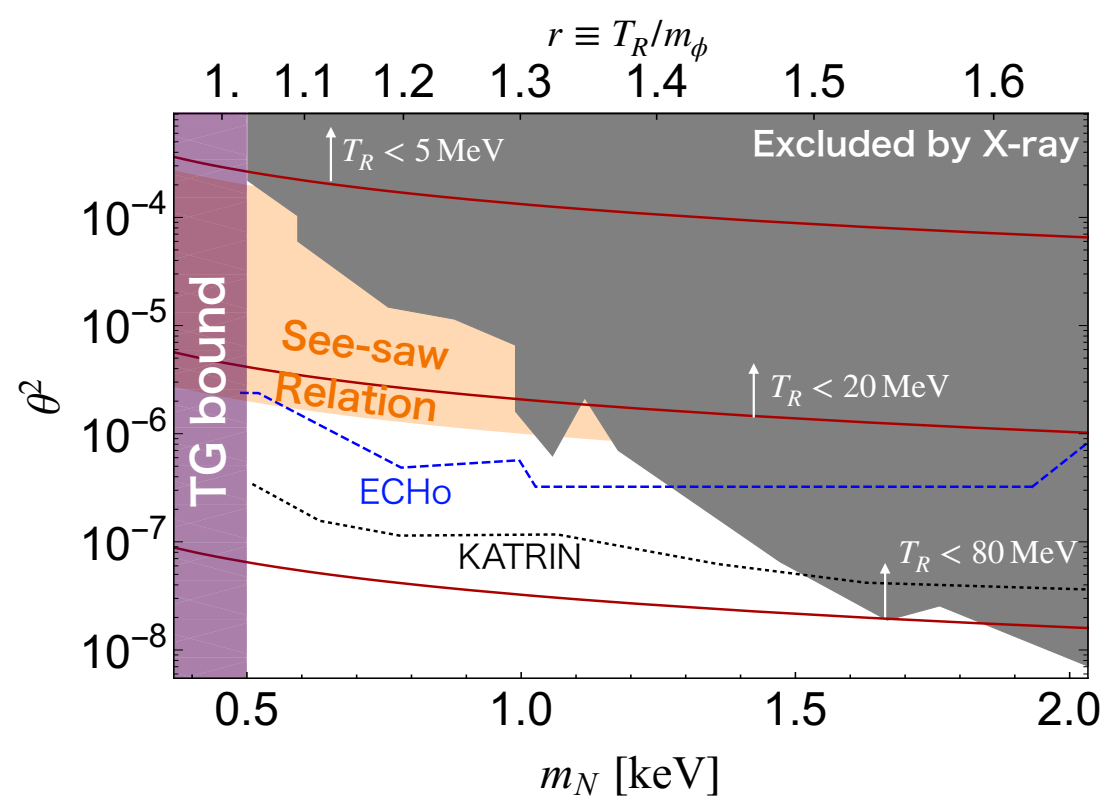

Figure 3. Parameter region for a Dirac sea DM of RHN in $m_{N^{-}} \theta^{2}$ plane. On the upper axis, we also show the predicted $r=T_{R} / m_{\phi}$ for the Dirac sea DM with $g=2, g_{\star}=g_{\star, s}=11$. Above the red solid lines, the reheating temperature should be smaller than $T_{R}=5 \mathrm{MeV}, 20 \mathrm{MeV}, 80 \mathrm{MeV}$.

shape of the deexcitation spectrum for ${ }^{163} \mathrm{Ho}$ by capturing an electron in ECHo [98] (see also HOLMES [99] and NuMECS [100] experiments) and the shape of the tritium $\beta$ decay spectrum in KATRIN [101-103] (see also Troitsk [104], Project 8 [105], and Ptolemy [106] experiments). When $\epsilon_{e}<1$, the sensitivity reaches on $\theta^{2}$ becomes worse by a factor of $\sim 1 / \epsilon_{e}^{2}$. Thus, the Dirac sea DM of the RHN with $M_{N} \sim 0.5-1 \mathrm{keV}$ can be a viable DM candidate, avoiding both the TG and $X$-ray bounds. ${ }^{10}$

In order for the Dirac sea RHN DM, the RHN should not be thermalized. The thermalization rate is estimated as $\Gamma_{\mathrm{th}}^{N}=\theta^{2} \Gamma_{\mathrm{th}}^{\nu}$, where $\Gamma_{\mathrm{th}}^{\nu} \sim G_{F}^{2} T^{5}$ is that of an active neutrino with $G_{F}$ being the Fermi constant. ${ }^{11}$ It is known that the active neutrino decouples from the thermal bath at the temperature below $\sim 1 \mathrm{MeV}$. Using the fact that the upper bound can be obtained by solving $H \sim \Gamma_{\text {th }}^{N}$, the upper bound from the non-thermalization is estimated as $T_{R} \lesssim 1 \mathrm{MeV} \times \theta^{-2 / 3}$. A more stringent bound comes from the thermal production of the RHN, whose abundance can be estimated as [107]

$$
\Omega_{N}^{\mathrm{th}} h^{2} \sim 0.11\left(\frac{\sin ^{2} 2 \theta}{10^{-3}}\right)\left(\frac{m_{N}}{1 \mathrm{keV}}\right)\left(\frac{T_{R}}{5 \mathrm{MeV}}\right)^{3} .
$$

Here again we assumed $\epsilon_{e}=1$. As previously noted, the thermal component of the RHN should not be dominant for $m_{N} \lesssim 10 \mathrm{keV}$ to evade the Lyman- $\alpha$ bound. In figure 3, we show the contours for $\Omega_{N}^{\text {th }} h^{2}=0.12 / 2$. Above the red solid lines to satisfy the condition,

\footnotetext{
${ }^{10}$ These bounds could be further alleviated if we have more than one RHN to be dominant DM.

${ }^{11}$ Notice that, when $r \gg 1$, there would be a further suppression of $1 / r$ for the scattering rate since the center-of-mass energy would be $\sim \sqrt{1 / r} T$. For the case of our interest, however, $r \sim 1$ and we neglect such a correction.
} 
the reheating temperature should satisfy $T_{R}=5,20,80 \mathrm{MeV}$ from the top to the bottom. Thus, even if the reheating temperature is much higher than $\sim 1 \mathrm{MeV}$ in order not to affect the BBN, we can avoid the overproduction of the RHN from the thermal production.

Interestingly, the Dirac sea RHN DM satisfying the see-saw relation not only can be searched for but also provide implication of the early Universe,

$$
O(1) \mathrm{MeV} \lesssim m_{\phi} \sim T_{R} \lesssim O(10) \mathrm{MeV}
$$

In particular with $\epsilon_{e}^{2}=O(0.1-1)$ the scenario can be tested in KATRIN and ECHo.

An interesting possibility of our scenario may be inflaton hunts [108-110]. In order for the present scenario, the inflaton should be as light as $1-100 \mathrm{MeV}$ and couple to SM particles for reheating. Therefore, it could be produced by current or future experiments. For instance, if we consider that the reheating is due to the interaction via inflaton mixing with the Higgs field, the inflaton with $m_{\phi} \lesssim 100 \mathrm{MeV}$ dominantly decays as $\phi \rightarrow e^{+} e^{-}$ at the rate of $\Gamma_{\phi \rightarrow e^{+} e^{-}}^{0} \sim \theta_{H}^{2} \frac{m_{e}^{2}}{16 \pi v^{2}} m_{\phi}$, with $\theta_{H}$ being the inflaton-Higgs mixing angle. Reheating temperature due to this decay process is estimated as ${ }^{12}$

$$
T_{R} \sim 5 \mathrm{MeV} \sqrt{\frac{m_{\phi}}{5 \mathrm{MeV}}}\left(\frac{\theta_{H}}{2 \times 10^{-4}}\right)\left(\frac{10}{g_{\star}}\right)^{1 / 4},
$$

from $3 H\left(T_{R}\right) \sim \Gamma_{\phi \rightarrow e^{+} e^{-}}$. This satisfies the constraint $\theta_{H}<3 \times 10^{-4}$ from the $K$ meson decays $[111,112]$ and may be searched for in the future experiments $[113,114]$.

\section{Reheating from evaporating inflaton}

In this section, we present an example of the model which gives rise to $r \gg 1$. During $t_{i} \lesssim t \lesssim t_{R}$, the reheating takes place with transferring the energy density of the inflaton to that of the SM particles. Here, in order for the reheating process, we consider the interaction between inflaton and SM particle with the following higher dimensional operator:

$$
\mathcal{L} \supset \frac{\phi}{\Lambda_{G}} G_{\mu \nu}^{(a)} G^{(a) \mu \nu},
$$

where $G_{\mu \nu}^{(a)}$ is the field strength of gluon and $\Lambda_{G}$ is the cut-off scale. This operator could be generated if heavy extra quarks couple to $\phi$. (If the extra quarks have quantum numbers of $\mathrm{SU}(2)_{L}$ and $\mathrm{U}(1)_{Y}$ gauge interactions, $\phi$ may also couple to those gauge fields. For simplicity, however, we adopt the interaction given above for our argument.) Then, the perturbative decay rate of $\phi$ in the vacuum is given by

$$
\Gamma_{\phi \rightarrow G G}^{(0)} \simeq \frac{2 m_{\phi}^{3}}{\pi \Lambda_{G}^{2}},
$$

where we have implicitly assumed that $m_{\phi}$ is heavier than the QCD scale,

$$
m_{\phi} \gtrsim 0.1 \mathrm{GeV}
$$

so that the non-perturbative effects from QCD dynamics is unimportant.

\footnotetext{
${ }^{12}$ Since $r \sim 1$, we can neglect the thermal back reaction discussed in section 5 .
} 
If the rate is large enough, the decay with a Bose-enhancement produces numerous gluons. Unlike the DM, the gluons have strong interaction and ambient plasma of the SM particle with temperature $T$ is soon formed. The enhancement is then disturbed by the thermal effect; it could be interpreted as the kinematical block due to the thermal mass of the gluon, which is of order $g_{s} T$. Assuming that the thermalization processes are so fast that the cosmic expansion is unimportant for its study, the energy density of the radiation, denoted as $\rho_{r}$, evolves as $\dot{\rho}_{r} \sim-\Gamma_{\phi \rightarrow G G}^{(0)} \rho_{\phi}$. Thus, the timescale relevant for the effects of the thermal blocking is estimated as

$$
t^{\text {(block) }} \sim \frac{\left.\rho_{r}\right|_{T \sim m_{\phi} / g_{s}}}{\Gamma_{\phi \rightarrow G G}^{(0)} \rho_{\phi}} .
$$

This timescale is shorter than the cosmic time if

$$
\Lambda_{G} \lesssim 1.3 \times 10^{14} \mathrm{GeV}\left(\frac{100 \mathrm{GeV}}{m_{\phi}}\right)^{1 / 2}\left(\frac{\rho_{\phi}^{1 / 4}}{100 \mathrm{GeV}}\right) .
$$

Even if the kinematical block occurs, the dissipation of $\phi$ may proceed due to the multiple scattering of $\phi$ in the thermal plasma [29, 115]. From the dimensional analysis, the dissipation rate is estimated as

$$
\Gamma_{\mathrm{dis}, G} \sim C \frac{T^{3}}{\Lambda_{G}^{2}}
$$

where $C$ is a numerical constant. In [115], $C$ is estimated from the imaginary part of the two point function of $\phi$ :

$$
C \sim \frac{\left(12 \pi \alpha_{s}\right)^{2}}{\log \left(1 / \alpha_{s}\right)}
$$

with $\alpha_{s}=\frac{g_{s}^{2}}{4 \pi}$. One can find that the dissipation effect becomes important at higher temperature. This implies that the reheating via the dissipation effect may become important soon after the inflation (preheating). As discussed in [115], the dissipation rate can hardly win the expansion rate of the universe if $\Lambda_{G} \sim M_{\mathrm{pl}}$. In the following, we consider the case that $\Lambda_{G} \ll M_{\mathrm{pl}}$ and the dissipation effect plays an important role in the reheating.

In discussing scenarios with dissipation, it should be noted that the dissipation rate is of the same order of the thermal production rate of the inflaton (as far as the cosmic temperature is higher than $m_{\phi}$ ); both of them are of the order of $\sim T^{3} / \Lambda_{G}^{2}$, by using the fact that the QCD coupling constant is sizable. Thus, if the dissipation rate becomes larger than the expansion rate of the universe, $\phi$ is likely to be thermalized. Such a thermally produced inflaton may affect the BBN processes in particular if the decay of the inflaton happens at the cosmic temperature of $\sim 1 \mathrm{MeV}$ or lower. (See [116-125] for the BBN bounds on long-lived particles.) In order that the thermally produced inflaton does not affect the standard BBN predictions, we require that the inflaton should disappear from the thermal bath well before the BBN epoch (i.e., conservatively, at $T \gtrsim 10 \mathrm{MeV}$ ). Such a 
requirement can be satisfied if

$$
\begin{aligned}
T_{\phi \text {-decay }} & \gtrsim 10 \mathrm{MeV}, \\
m_{\phi} & \gtrsim 10 \mathrm{MeV} .
\end{aligned}
$$

Here, $T_{\phi \text {-decay }}$ is the cosmic temperature just after the decay of the inflaton (assuming that the inflaton decays after becoming non-relativistic); $T_{\phi \text {-decay }}$ is estimated by solving $3 H\left(T_{\phi \text {-decay }}\right) \sim \Gamma_{\phi \rightarrow G G}$, and is obtained as

$$
T_{\phi \text {-decay }} \sim\left(\frac{g_{\star} \pi^{2}}{90}\right)^{-1 / 4} \sqrt{\Gamma_{\phi \rightarrow G G} M_{\mathrm{pl}} / 3} .
$$

Note that the thermally produced inflaton may dominate the Universe. In such a case, $T_{\phi \text {-decay }}$ is regarded as the reheating temperature due to the decay of such inflaton.

We can check numerically whether the reheating is successful. We solve the following set of Boltzmann equations:

$$
\begin{aligned}
\dot{\rho}_{\phi}+3 H \rho_{\phi} & =-\left(\Gamma_{\phi \rightarrow G G}^{(T)}+\Gamma_{\mathrm{dis}, \mathrm{G}}\right) \rho_{\phi}, \\
\dot{\rho}_{r}+4 H \rho_{r} & =\left(\Gamma_{\phi \rightarrow G G}^{(T)}+\Gamma_{\mathrm{dis}, \mathrm{G}}\right) \rho_{\phi},
\end{aligned}
$$

where $\Gamma_{\phi \rightarrow G G}^{(T)}$ is the decay rate of $\phi$ in thermal bath. The detailed study of the effects of the thermal blocking is beyond the scope of this paper. We expect that the perturbative decay proceeds at least until the cosmic temperature becomes as large as $\sim m_{\phi} / g_{s}$. Then, for $T \gtrsim m_{\phi} / g_{s}$, the perturbative decay of $\phi$ is suppressed because of the thermal blocking. As an approximated procedure, in our numerical analysis, we solve the above set of Boltzmann equations with the initial condition of $\rho_{r}=C g_{\star} \pi^{2} T^{4} /\left.30\right|_{T=m_{\phi} / g_{s}}$ (with $C$ being a constant of $O(0.1-1)$ ) and $\rho_{\phi}=g_{\star} \pi^{2} T_{R}^{4} / 30$, with setting $\Gamma_{\phi \rightarrow G G}^{(T)}=0$ (assuming that the perturbative decay is maximally blocked for $\left.\rho_{r}>C g_{\star} \pi^{2} T^{4} /\left.30\right|_{T=m_{\phi} / g_{s}}\right)$. In our analysis, we concentrate on the case that the narrow resonance condition for the gluon production process holds, leaving the study of the case of the broad resonance for a future work. Such a condition is given by $q^{\prime} \equiv \frac{\bar{\phi}}{\Lambda_{G}} \lesssim 1$. Requiring $q^{\prime} \lesssim 1$ at the time of the reheating, we obtain

$$
\Lambda_{G} \gtrsim 10^{10} \mathrm{GeV} \times\left(\frac{r}{10^{5}}\right)^{2}\left(\frac{m_{\phi}}{1 \mathrm{GeV}}\right) .
$$

In this regime, the expansion rate during the inflation $H_{\text {inf }}$ may satisfy $\Gamma_{\text {dis, } G} \gtrsim H_{\text {inf }}$, with which a successful instantaneous reheating occurs.

We numerically solve the set of Boltzmann equations (5.11) and (5.12) and find that the energy density of $\phi$ can be efficiently converted to that of radiation for some region of the parameter space. In figure 4 , we show the maximal value of $r, r_{\max }$, as well as the corresponding $m_{\phi}$ and $T_{R}$ by varying $\Lambda_{G}$. The lines satisfy the inequalities (3.1), (5.3), (5.5), (5.8), and (5.13). In the present model, we find that $r$ can be as large as $10^{5}-10^{6}$. We see that the slope suddenly changes at around $\Lambda_{G}=10^{9} \mathrm{GeV}$ and $10^{11-12} \mathrm{GeV}$. For $\Lambda_{G}<10^{9} \mathrm{GeV}$ $\left(10^{9} \mathrm{GeV}<\Lambda_{G}<10^{11-12} \mathrm{GeV}, \Lambda_{G}>10^{11-12} \mathrm{GeV}\right), r_{\max }$ is determined by the constraints (5.3) and (5.8) ((5.8) and (5.13), (3.1) and (5.13)). The constraint (5.5) is less 


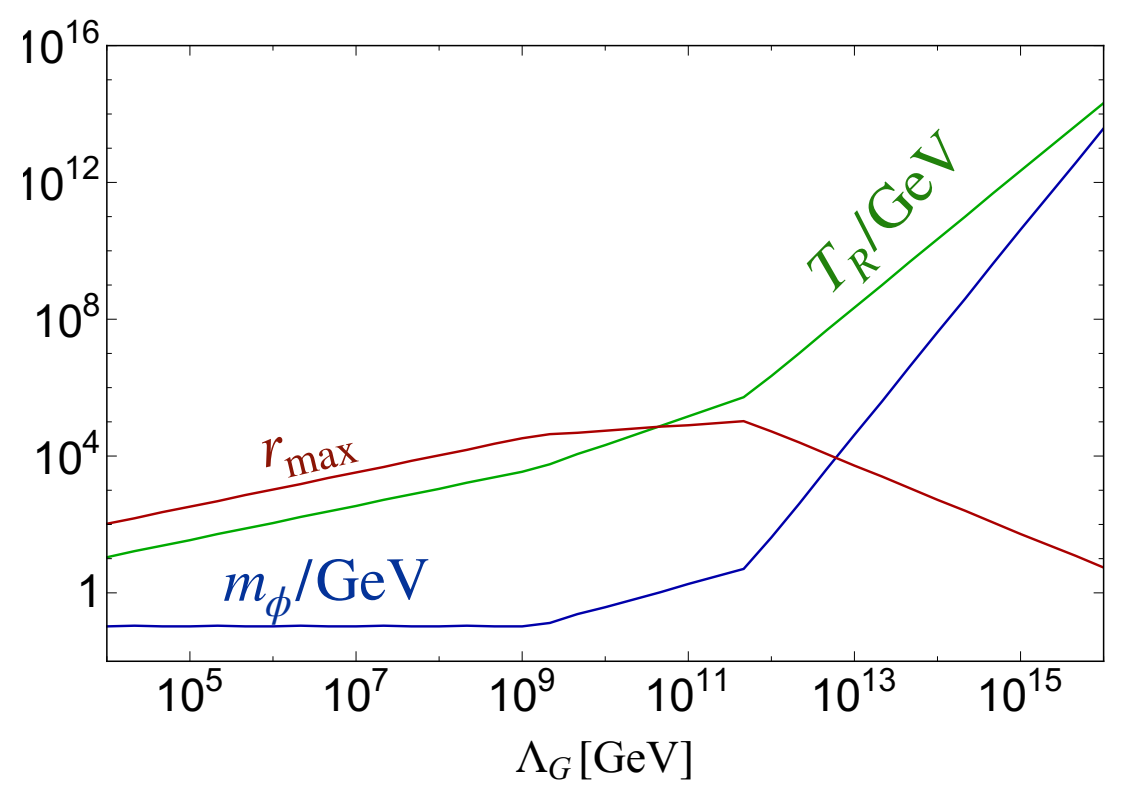

Figure 4. The maximal $r$ by varying $\Lambda_{G}$ [red line]. The line satisfies the inequalities of (3.1), (5.5), (5.3), (5.8), and (5.13). The corresponding reheating temperature and inflaton mass are also shown in green and blue solid lines, respectively.

stringent than the others in the whole range. The maximal value of $r$ can be obtained when $\Lambda_{G} \sim 10^{10-11} \mathrm{GeV}, T_{\phi} \sim 10 \mathrm{MeV}$ and $\bar{\phi} / \Lambda_{G} \sim 1$. We have checked that the result is insensitive to the choice of $C$ as far as $C \sim 0.1-1$. Thus, the reheating temperature higher than the inflaton mass is possible in the present scenario.

As we mentioned, the thermally produced inflaton may dominate the Universe subsequently and then decay. The DM velocity is further suppressed by the extra dilution due to the decay of the thermally produced inflaton. The dilution factor $\Delta$ is estimated as $\Delta \sim T_{\phi \text {-decay }} / m_{\phi}$ and, numerically,

$$
\Delta^{1 / 3} \sim 0.2 \times\left(\frac{m_{\phi}}{1 \mathrm{GeV}}\right)^{1 / 6}\left(\frac{10}{g_{\star}}\right)^{1 / 12}\left(\frac{10^{11} \mathrm{GeV}}{\Lambda_{G}}\right)^{1 / 3} .
$$

With the effect of the extra dilution due to the decay of thermally produced inflaton, the Lyman- $\alpha$ bound in figure 1 can be alleviated and $m_{\chi} \sim 10^{-4} \mathrm{eV}$ may become possible without conflicting the Lyman- $\alpha$ constraint. We also emphasize that, although an extra entropy production can make the DM colder, we still need $r \gtrsim 1$ to make the DM number density large enough.

Again, from eq. (2.18) and $H \sim q^{2} m_{\phi}$, we need $q m_{\phi} \gtrsim \Gamma_{\text {dis }, G}$ for the consistency of the DM production, which implies

$$
q \gtrsim 10^{-12}\left(\frac{m_{\phi}}{1 \mathrm{GeV}}\right)^{2}\left(\frac{10^{11} \mathrm{GeV}}{\Lambda_{G}}\right)^{2}\left(\frac{r}{10^{3}}\right)^{3} .
$$

For the axion DM, we have $q \sim \bar{\phi} / \Lambda_{a} \sim 10^{-3} q^{\prime}$ for $\Lambda_{a} / \Lambda_{G} \sim 10^{3}$. Thus, this can be easily satisfied. 


\section{Conclusions and discussion}

We have studied the DM production due to the decay of the inflaton (or more generically, an oscillating scalar field). If the inflaton coupling to the SM sector has a sizable strength, the reheating temperature due to the inflaton decay can be comparable to or higher than the inflaton mass. If $T_{R} \gtrsim m_{\phi}$, then the DM produced by the inflaton has a momentum smaller than those of particles in the thermal bath (consisting of the SM particles), assuming that the interaction of the DM with the SM particles or itself is negligibly weak. It can help the DM produced by the decay to be cold enough to be consistent with the Lyman- $\alpha$ bound on warm DMs even if the DM mass is smaller than $O(1) \mathrm{keV}$.

We have also shown that, if the DM is bosonic, the production of the DM from the inflaton decay can be enhanced due to the effect of the stimulated emission, like the LASER. The mechanism, called DASER (i.e, the DM amplification by stimulated emotion of radiation), can significantly enhance the DM abundance and can make light bosonic DM scenarios viable. In addition, if the DM is fermionic, the DM produced by the decay may form Dirac sea. The DASER and Dirac sea DMs produced by the inflaton decay may be searched for by future observations of the $21 \mathrm{~cm}$ lines. The DMs produced by the above mentioned mechanism have null isocurvature perturbations.

Finally, we comment that the DMs produced in the present scenarios may have very special momentum distribution, and the information about the production mechanism discussed here may be embedded in the momentum distribution of the DM. For example, the momentum of the bosonic DM produced by the DASER mechanism would have sharp peak at the IR mode at the momentum $\sim m_{\phi} / a\left[t_{i}\right]$. From a quantum field theory approach, the peak width can be approximated as $\sim \sqrt{m_{\phi} H\left(t_{i}\right)} / a\left(t_{i}\right)$ (see [41]). By combining the two, we can get $m_{\phi} / H\left(t_{i}\right)$. In addition, momentum distribution of the modes produced during the preheating depends on the thermal history during the preheating. One may probe the reheating phase (see also ref. [126]) or preheating phase if the information about the momentum distribution of the DM becomes available. For this purpose, further study of the structure formation with non-standard momentum distribution of the DM is needed.

\section{Acknowledgments}

This work is supported by JSPS KAKENHI grant Nos. 16H06490 (TM and WY) and $18 \mathrm{~K} 03608$ (TM). 
Open Access. This article is distributed under the terms of the Creative Commons Attribution License (CC-BY 4.0), which permits any use, distribution and reproduction in any medium, provided the original author(s) and source are credited.

\section{References}

[1] I.G. Irastorza and J. Redondo, New experimental approaches in the search for axion-like particles, Prog. Part. Nucl. Phys. 102 (2018) 89 [arXiv:1801.08127] [InSPIRE].

[2] PandaX collaboration, Dark matter direct search sensitivity of the PandaX-4T experiment, Sci. China Phys. Mech. Astron. 62 (2019) 31011 [arXiv:1806.02229] [InSPIRE].

[3] LZ collaboration, The LUX-ZEPLIN (LZ) Experiment, Nucl. Instrum. Meth. A 953 (2020) 163047 [arXiv: 1910.09124] [INSPIRE].

[4] XENON collaboration, Excess electronic recoil events in XENON1T, Phys. Rev. D 102 (2020) 072004 [arXiv: 2006.09721] [INSPIRE].

[5] M. Viel, J. Lesgourgues, M.G. Haehnelt, S. Matarrese and A. Riotto, Constraining warm dark matter candidates including sterile neutrinos and light gravitinos with WMAP and the Lyman-alpha forest, Phys. Rev. D 71 (2005) 063534 [astro-ph/0501562] [INSPIRE].

[6] V. Iršič et al., New Constraints on the free-streaming of warm dark matter from intermediate and small scale Lyman- $\alpha$ forest data, Phys. Rev. D 96 (2017) 023522 [arXiv: 1702.01764] [INSPIRE].

[7] A. Kamada and K. Yanagi, Constraining FIMP from the structure formation of the Universe: analytic mapping from $m_{\mathrm{WDM}}$, JCAP 11 (2019) 029 [arXiv: 1907.04558] [INSPIRE].

[8] J. Preskill, M.B. Wise and F. Wilczek, Cosmology of the Invisible Axion, Phys. Lett. B 120 (1983) 127 [INSPIRE].

[9] L.F. Abbott and P. Sikivie, A Cosmological Bound on the Invisible Axion, Phys. Lett. B 120 (1983) 133 [INSPIRE].

[10] M. Dine and W. Fischler, The Not So Harmless Axion, Phys. Lett. B 120 (1983) 137 [INSPIRE].

[11] P.W. Graham, J. Mardon and S. Rajendran, Vector Dark Matter from Inflationary Fluctuations, Phys. Rev. D 93 (2016) 103520 [arXiv:1504.02102] [InSPIRE].

[12] Y. Ema, K. Nakayama and Y. Tang, Production of Purely Gravitational Dark Matter: The Case of Fermion and Vector Boson, JHEP 07 (2019) 060 [arXiv:1903.10973] [INSPIRE].

[13] P.W. Graham and A. Scherlis, Stochastic axion scenario, Phys. Rev. D 98 (2018) 035017 [arXiv: 1805.07362] [INSPIRE].

[14] F. Takahashi, W. Yin and A.H. Guth, QCD axion window and low-scale inflation, Phys. Rev. D 98 (2018) 015042 [arXiv: 1805.08763] [INSPIRE].

[15] N. Kitajima, T. Sekiguchi and F. Takahashi, Cosmological abundance of the QCD axion coupled to hidden photons, Phys. Lett. B 781 (2018) 684 [arXiv:1711.06590] [INSPIRE].

[16] P. Agrawal, N. Kitajima, M. Reece, T. Sekiguchi and F. Takahashi, Relic Abundance of Dark Photon Dark Matter, Phys. Lett. B 801 (2020) 135136 [arXiv:1810. 07188] [INSPIRE]. 
[17] R.T. Co, A. Pierce, Z. Zhang and Y. Zhao, Dark Photon Dark Matter Produced by Axion Oscillations, Phys. Rev. D 99 (2019) 075002 [arXiv:1810.07196] [InSPIRE].

[18] J.A. Dror, K. Harigaya and V. Narayan, Parametric Resonance Production of Ultralight Vector Dark Matter, Phys. Rev. D 99 (2019) 035036 [arXiv:1810.07195] [INSPIRE].

[19] P. Agrawal, G. Marques-Tavares and W. Xue, Opening up the QCD axion window, JHEP 03 (2018) 049 [arXiv: 1708.05008] [INSPIRE].

[20] A. Mazumdar and S. Qutub, Nonperturbative overproduction of axionlike particles via derivative interactions, Phys. Rev. D 93 (2016) 043502 [arXiv: 1508.04136] [INSPIRE].

[21] R.T. Co, L.J. Hall and K. Harigaya, QCD Axion Dark Matter with a Small Decay Constant, Phys. Rev. Lett. 120 (2018) 211602 [arXiv:1711.10486] [InSPIRE].

[22] L. Randall, J. Scholtz and J. Unwin, Flooded Dark Matter and S Level Rise, JHEP 03 (2016) 011 [arXiv: 1509.08477] [InSPIRE].

[23] J. Yokoyama, Can oscillating scalar fields decay into particles with a large thermal mass?, Phys. Lett. B 635 (2006) 66 [hep-ph/0510091] [INSPIRE].

[24] A. Anisimov, W. Buchmüller, M. Drewes and S. Mendizabal, Nonequilibrium Dynamics of Scalar Fields in a Thermal Bath, Annals Phys. 324 (2009) 1234 [arXiv:0812.1934] [INSPIRE].

[25] M. Drewes, On the Role of Quasiparticles and thermal Masses in Nonequilibrium Processes in a Plasma, arXiv: 1012.5380 [INSPIRE].

[26] K. Mukaida and K. Nakayama, Dynamics of oscillating scalar field in thermal environment, JCAP 01 (2013) 017 [arXiv:1208.3399] [INSPIRE].

[27] M. Drewes and J.U. Kang, The Kinematics of Cosmic Reheating, Nucl. Phys. B 875 (2013) 315 [Erratum ibid. 888 (2014) 284] [arXiv:1305.0267] [INSPIRE].

[28] K. Mukaida and K. Nakayama, Dissipative Effects on Reheating after Inflation, JCAP 03 (2013) 002 [arXiv: 1212.4985] [INSPIRE].

[29] T. Moroi, K. Mukaida, K. Nakayama and M. Takimoto, Axion Models with High Scale Inflation, JHEP 11 (2014) 151 [arXiv:1407.7465] [INSPIRE].

[30] T. Moroi, M. Yamaguchi and T. Yanagida, On the solution to the Polonyi problem with O(10 TeV) gravitino mass in supergravity, Phys. Lett. B 342 (1995) 105 [hep-ph/9409367] [INSPIRE].

[31] M. Kawasaki, T. Moroi and T. Yanagida, Constraint on the reheating temperature from the decay of the Polonyi field, Phys. Lett. B 370 (1996) 52 [hep-ph/9509399] [InSPIRE].

[32] T. Moroi and L. Randall, Wino cold dark matter from anomaly mediated SUSY breaking, Nucl. Phys. B 570 (2000) 455 [hep-ph/9906527] [INSPIRE].

[33] K.S. Jeong, M. Shimosuka and M. Yamaguchi, Light Higgsino in Heavy Gravitino Scenario with Successful Electroweak Symmetry Breaking, JHEP 09 (2012) 050 [arXiv:1112.5293] [INSPIRE].

[34] J. Ellis, M.A.G. Garcia, D.V. Nanopoulos, K.A. Olive and M. Peloso, Post-Inflationary Gravitino Production Revisited, JCAP 03 (2016) 008 [arXiv:1512.05701] [InSPIRE].

[35] K. Harigaya, M. Kawasaki, K. Mukaida and M. Yamada, Dark Matter Production in Late Time Reheating, Phys. Rev. D 89 (2014) 083532 [arXiv:1402.2846] [InSPIRE]. 
[36] M.A.G. Garcia and M.A. Amin, Prethermalization production of dark matter, Phys. Rev. D 98 (2018) 103504 [arXiv: 1806.01865] [INSPIRE].

[37] K. Harigaya, K. Mukaida and M. Yamada, Dark Matter Production during the Thermalization Era, JHEP 07 (2019) 059 [arXiv: 1901.11027] [INSPIRE].

[38] M.A.G. Garcia, K. Kaneta, Y. Mambrini and K.A. Olive, Reheating and Post-inflationary Production of Dark Matter, Phys. Rev. D 101 (2020) 123507 [arXiv: 2004.08404] [INSPIRE].

[39] D.J.H. Chung, E.W. Kolb and A. Riotto, Superheavy dark matter, Phys. Rev. D 59 (1998) 023501 [hep-ph/9802238] [inSPIRE].

[40] D.J.H. Chung, E.W. Kolb and A. Riotto, Nonthermal supermassive dark matter, Phys. Rev. Lett. 81 (1998) 4048 [hep-ph/9805473] [INSPIRE].

[41] T. Moroi and W. Yin, Particle Production from Oscillating Scalar Field and Consistency of Boltzmann Equation, arXiv:2011.12285 [INSPIRE].

[42] E.W. Kolb and M.S. Turner, The Early Universe, Front. Phys. 69 (1990) 1 [InSPIRE].

[43] L. Kofman, A.D. Linde and A.A. Starobinsky, Reheating after inflation, Phys. Rev. Lett. 73 (1994) 3195 [hep-th/9405187] [INSPIRE].

[44] L. Kofman, A.D. Linde and A.A. Starobinsky, Towards the theory of reheating after inflation, Phys. Rev. D 56 (1997) 3258 [hep-ph/9704452] [INSPIRE].

[45] J.F. Dufaux, G.N. Felder, L. Kofman, M. Peloso and D. Podolsky, Preheating with trilinear interactions: Tachyonic resonance, JCAP 07 (2006) 006 [hep-ph/0602144] [INSPIRE].

[46] M.A. Amin, J. Fan, K.D. Lozanov and M. Reece, Cosmological dynamics of Higgs potential fine tuning, Phys. Rev. D 99 (2019) 035008 [arXiv:1802.00444] [InSPIRE].

[47] S. Matsumoto and T. Moroi, Decay of scalar condensation in quantum field theory, Phys. Rev. D 77 (2008) 045014 [arXiv: 0709.4338] [INSPIRE].

[48] E.D. Carlson, M.E. Machacek and L.J. Hall, Self-interacting dark matter, Astrophys. J. 398 (1992) 43 [INSPIRE].

[49] S.Y. Khlebnikov and I.I. Tkachev, Classical decay of inflaton, Phys. Rev. Lett. 77 (1996) 219 [hep-ph/9603378] [INSPIRE].

[50] R. Micha and I.I. Tkachev, Relativistic turbulence: A Long way from preheating to equilibrium, Phys. Rev. Lett. 90 (2003) 121301 [hep-ph/0210202] [InSPIRE].

[51] R. Micha and I.I. Tkachev, Turbulent thermalization, Phys. Rev. D 70 (2004) 043538 [hep-ph/0403101] [INSPIRE].

[52] R. Daido, F. Takahashi and W. Yin, The ALP miracle: unified inflaton and dark matter, JCAP 05 (2017) 044 [arXiv: 1702.03284] [INSPIRE].

[53] R. Daido, F. Takahashi and W. Yin, The ALP miracle revisited, JHEP 02 (2018) 104 [arXiv: 1710.11107] [INSPIRE].

[54] S.-M. Choi, Y.-J. Kang, H.M. Lee and K. Yamashita, Unitary inflaton as decaying dark matter, JHEP 05 (2019) 060 [arXiv:1902.03781] [INSPIRE].

[55] F. Takahashi, M. Yamada and W. Yin, What if ALP dark matter for the XENON1T excess is the inflaton, JHEP 01 (2021) 152 [arXiv:2007.10311] [INSPIRE]. 
[56] S. Tremaine and J.E. Gunn, Dynamical Role of Light Neutral Leptons in Cosmology, Phys. Rev. Lett. 42 (1979) 407 [INSPIRE].

[57] A. Boyarsky, O. Ruchayskiy and D. Iakubovskyi, A Lower bound on the mass of Dark Matter particles, JCAP 03 (2009) 005 [arXiv: 0808.3902] [INSPIRE].

[58] L. Randall, J. Scholtz and J. Unwin, Cores in Dwarf Galaxies from Fermi Repulsion, Mon. Not. Roy. Astron. Soc. 467 (2017) 1515 [arXiv:1611.04590] [InSPIRE].

[59] M. Sitwell, A. Mesinger, Y.-Z. Ma and K. Sigurdson, The Imprint of Warm Dark Matter on the Cosmological 21-cm Signal, Mon. Not. Roy. Astron. Soc. 438 (2014) 2664 [arXiv:1310.0029] [INSPIRE].

[60] G.N. Felder, J. García-Bellido, P.B. Greene, L. Kofman, A.D. Linde and I. Tkachev, Dynamics of symmetry breaking and tachyonic preheating, Phys. Rev. Lett. 87 (2001) 011601 [hep-ph/0012142] [INSPIRE].

[61] G.N. Felder, L. Kofman and A.D. Linde, Tachyonic instability and dynamics of spontaneous symmetry breaking, Phys. Rev. D 64 (2001) 123517 [hep-th/0106179] [INSPIRE].

[62] P. Brax, J.-F. Dufaux and S. Mariadassou, Preheating after Small-Field Inflation, Phys. Rev. D 83 (2011) 103510 [arXiv: 1012.4656] [INSPIRE].

[63] J.H. Chang, R. Essig and S.D. McDermott, Supernova 1987 A Constraints on Sub-GeV Dark Sectors, Millicharged Particles, the QCD Axion, and an Axion-like Particle, JHEP 09 (2018) 051 [arXiv: 1803.00993] [inSPIRE].

[64] R. Mayle, J.R. Wilson, J.R. Ellis, K.A. Olive, D.N. Schramm and G. Steigman, Constraints on Axions from SN 1987a, Phys. Lett. B 203 (1988) 188 [inSPIRE].

[65] G. Raffelt and D. Seckel, Bounds on Exotic Particle Interactions from SN 1987a, Phys. Rev. Lett. 60 (1988) 1793 [INSPIRE].

[66] K.J. Bae, J.-H. Huh and J.E. Kim, Update of axion CDM energy, JCAP 09 (2008) 005 [arXiv: 0806.0497] [INSPIRE].

[67] L. Visinelli and P. Gondolo, Dark Matter Axions Revisited, Phys. Rev. D 80 (2009) 035024 [arXiv: 0903.4377] [INSPIRE].

[68] D.H. Lyth, Axions and inflation: Sitting in the vacuum, Phys. Rev. D 45 (1992) 3394 [INSPIRE].

[69] T. Kobayashi, R. Kurematsu and F. Takahashi, Isocurvature Constraints and Anharmonic Effects on QCD Axion Dark Matter, JCAP 09 (2013) 032 [arXiv:1304.0922] [INSPIRE].

[70] F. Takahashi and W. Yin, QCD axion on hilltop by a phase shift of $\pi$, JHEP 10 (2019) 120 [arXiv: 1908.06071] [INSPIRE].

[71] MADMAX Working Group collaboration, Dielectric Haloscopes: A New Way to Detect Axion Dark Matter, Phys. Rev. Lett. 118 (2017) 091801 [arXiv:1611. 05865] [INSPIRE].

[72] MADMAX collaboration, A new experimental approach to probe QCD axion dark matter in the mass range above $40 \mu \mathrm{eV}$, Eur. Phys. J. C 79 (2019) 186 [arXiv:1901.07401] [INSPIRE].

[73] B.T. McAllister, G. Flower, E.N. Ivanov, M. Goryachev, J. Bourhill and M.E. Tobar, The ORGAN Experiment: An axion haloscope above 15 GHz, Phys. Dark Univ. 18 (2017) 67 [arXiv: 1706. 00209] [INSPIRE]. 
[74] D.J.E. Marsh, K.-C. Fong, E.W. Lentz, L. Smejkal and M.N. Ali, Proposal to Detect Dark Matter using Axionic Topological Antiferromagnets, Phys. Rev. Lett. 123 (2019) 121601 [arXiv: 1807.08810] [INSPIRE].

[75] S. Chigusa, T. Moroi and K. Nakayama, Detecting light boson dark matter through conversion into a magnon, Phys. Rev. D 101 (2020) 096013 [arXiv:2001.10666] [InSPIRE].

[76] I.G. Irastorza et al., Towards a new generation axion helioscope, JCAP 06 (2011) 013 [arXiv:1103.5334] [INSPIRE].

[77] E. Armengaud et al., Conceptual Design of the International Axion Observatory (IAXO), 2014 JINST 9 T05002 [arXiv:1401.3233] [INSPIRE].

[78] IAXO collaboration, Physics potential of the International Axion Observatory (IAXO), JCAP 06 (2019) 047 [arXiv: 1904.09155] [InSPIRE].

[79] A. Arvanitaki and A.A. Geraci, Resonantly Detecting Axion-Mediated Forces with Nuclear Magnetic Resonance, Phys. Rev. Lett. 113 (2014) 161801 [arXiv:1403.1290] [INSPIRE].

[80] ARIADNE collaboration, Progress on the ARIADNE axion experiment, Springer Proc. Phys. 211 (2018) 151 [arXiv:1710.05413] [InSPIRE].

[81] L.D. McLerran, E. Mottola and M.E. Shaposhnikov, Sphalerons and Axion Dynamics in High Temperature QCD, Phys. Rev. D 43 (1991) 2027 [InSPIRE].

[82] F. Takahashi, M. Yamada and W. Yin, XENON1T Excess from Anomaly-Free Axionlike Dark Matter and Its Implications for Stellar Cooling Anomaly, Phys. Rev. Lett. 125 (2020) 161801 [arXiv:2006.10035] [INSPIRE].

[83] G. Alonso-Álvarez, F. Ertas, J. Jaeckel, F. Kahlhoefer and L.J. Thormaehlen, Hidden Photon Dark Matter in the Light of XENON1T and Stellar Cooling, JCAP 11 (2020) 029 [arXiv: 2006.11243] [INSPIRE].

[84] P. Athron et al., Global fits of axion-like particles to XENON1T and astrophysical data, arXiv:2007.05517 [INSPIRE].

[85] I.M. Bloch, A. Caputo, R. Essig, D. Redigolo, M. Sholapurkar and T. Volansky, Exploring new physics with $O(\mathrm{keV})$ electron recoils in direct detection experiments, JHEP 01 (2021) 178 [arXiv: 2006.14521] [INSPIRE].

[86] K. Nakayama and Y. Tang, Gravitational Production of Hidden Photon Dark Matter in Light of the XENON1T Excess, Phys. Lett. B 811 (2020) 135977 [arXiv:2006.13159] [INSPIRE].

[87] H. An, M. Pospelov, J. Pradler and A. Ritz, New limits on dark photons from solar emission and keV scale dark matter, Phys. Rev. D 102 (2020) 115022 [arXiv: 2006.13929] [InSPIRE].

[88] T. Li, The KSVZ Axion and Pseudo-Nambu-Goldstone Boson Models for the XENON1T Excess, arXiv:2007.00874 [INSPIRE].

[89] P. Minkowski, $\mu \rightarrow$ er at a Rate of One Out of $10^{9}$ Muon Decays?, Phys. Lett. B 67 (1977) 421 [INSPIRE].

[90] T. Yanagida, Horizontal gauge symmetry and masses of neutrinos, Conf. Proc. C $\mathbf{7 9 0 2 1 3 1}$ (1979) 95 [INSPIRE].

[91] S.L. Glashow, The Future of Elementary Particle Physics, NATO Sci. Ser. B 61 (1980) 687 [INSPIRE]. 
[92] M. Gell-Mann, P. Ramond and R. Slansky, Complex Spinors and Unified Theories, Conf. Proc. C 790927 (1979) 315 [arXiv: 1306.4669] [InSPIRE].

[93] R.N. Mohapatra and G. Senjanović, Neutrino Mass and Spontaneous Parity Nonconservation, Phys. Rev. Lett. 44 (1980) 912 [InSPIRE].

[94] Particle Data Group collaboration, Review of Particle Physics, PTEP 2020 (2020) 083C01 [INSPIRE].

[95] T. Asaka, S. Blanchet and M. Shaposhnikov, The nuMSM, dark matter and neutrino masses, Phys. Lett. B 631 (2005) 151 [hep-ph/0503065] [InSPIRE].

[96] T. Asaka and M. Shaposhnikov, The $\nu M S M$, dark matter and baryon asymmetry of the universe, Phys. Lett. B 620 (2005) 17 [hep-ph/0505013] [INSPIRE].

[97] M. Drewes et al., A White Paper on keV Sterile Neutrino Dark Matter, JCAP 01 (2017) 025 [arXiv: 1602.04816] [INSPIRE].

[98] P.E. Filianin et al., On the keV sterile neutrino search in electron capture, J. Phys. G 41 (2014) 095004 [arXiv: 1402.4400] [INSPIRE].

[99] B. Alpert et al., HOLMES - The Electron Capture Decay of ${ }^{163}$ Ho to Measure the Electron Neutrino Mass with sub-eV sensitivity, Eur. Phys. J. C 75 (2015) 112 [arXiv:1412.5060] [INSPIRE].

[100] J.W. Engle, E.R. Birnbaum, H.R. Trellue, K.D. John, M.W. Rabin and F.M. Nortier, Evaluation of ${ }^{163}$ Ho production options for neutrino mass measurements with microcalorimeter detectors, Nucl. Instrum. Meth. B 311 (2013) 131 [INSPIRE].

[101] KATRIN collaboration, KATRIN design report 2004, FZKA-7090 (2005) [INSPIRE].

[102] S. Mertens et al., Sensitivity of Next-Generation Tritium Beta-Decay Experiments for keV-Scale Sterile Neutrinos, JCAP 02 (2015) 020 [arXiv: 1409.0920] [INSPIRE].

[103] S. Mertens et al., Wavelet approach to search for sterile neutrinos in tritium $\beta$-decay spectra, Phys. Rev. D 91 (2015) 042005 [arXiv:1410.7684] [INSPIRE].

[104] C. Kraus et al., Final results from phase II of the Mainz neutrino mass search in tritium beta decay, Eur. Phys. J. C 40 (2005) 447 [hep-ex/0412056] [INSPIRE].

[105] Project 8 collaboration, Single electron detection and spectroscopy via relativistic cyclotron radiation, Phys. Rev. Lett. 114 (2015) 162501 [arXiv:1408.5362] [InSPIRE].

[106] S. Betts et al., Development of a Relic Neutrino Detection Experiment at PTOLEMY: Princeton Tritium Observatory for Light, Early-Universe, Massive-Neutrino Yield, in Community Summer Study 2013: Snowmass on the Mississippi, (2013) [arXiv:1307.4738] [INSPIRE].

[107] G. Gelmini, S. Palomares-Ruiz and S. Pascoli, Low reheating temperature and the visible sterile neutrino, Phys. Rev. Lett. 93 (2004) 081302 [astro-ph/0403323] [INSPIRE].

[108] F. Bezrukov and D. Gorbunov, Light inflaton Hunter's Guide, JHEP 05 (2010) 010 [arXiv: 0912.0390] [INSPIRE].

[109] F. Takahashi and W. Yin, ALP inflation and Big Bang on Earth, JHEP 07 (2019) 095 [arXiv: 1903.00462] [INSPIRE].

[110] N. Okada and D. Raut, Hunting Inflaton at FASER, arXiv:1910.09663 [INSPIRE]. 
[111] BNL-E949 collaboration, Study of the decay $K^{+} \rightarrow \pi^{+} \nu \bar{\nu}$ in the momentum region $140<P_{\pi}<199 \mathrm{MeV} / c$, Phys. Rev. D 79 (2009) 092004 [arXiv:0903.0030] [InSPIRE].

[112] M.W. Winkler, Decay and detection of a light scalar boson mixing with the Higgs boson, Phys. Rev. D 99 (2019) 015018 [arXiv:1809.01876] [INSPIRE].

[113] B. Batell, J. Berger and A. Ismail, Probing the Higgs Portal at the Fermilab Short-Baseline Neutrino Experiments, Phys. Rev. D 100 (2019) 115039 [arXiv:1909.11670] [INSPIRE].

[114] NA62 collaboration, An investigation of the very rare $K^{+} \rightarrow \pi^{+} \nu \bar{\nu}$ decay, JHEP 11 (2020) 042 [arXiv: 2007.08218] [INSPIRE].

[115] M. Laine, On bulk viscosity and moduli decay, Prog. Theor. Phys. Suppl. 186 (2010) 404 [arXiv:1007.2590] [INSPIRE].

[116] M. Kawasaki, K. Kohri and N. Sugiyama, Cosmological constraints on late time entropy production, Phys. Rev. Lett. 82 (1999) 4168 [astro-ph/9811437] [INSPIRE].

[117] M. Kawasaki, K. Kohri and N. Sugiyama, MeV scale reheating temperature and thermalization of neutrino background, Phys. Rev. D 62 (2000) 023506 [astro-ph/0002127] [INSPIRE].

[118] S. Hannestad, What is the lowest possible reheating temperature?, Phys. Rev. D 70 (2004) 043506 [astro-ph/0403291] [INSPIRE].

[119] K. Ichikawa, M. Kawasaki and F. Takahashi, Constraint on the Effective Number of Neutrino Species from the WMAP and SDSS LRG Power Spectra, JCAP 05 (2007) 007 [astro-ph/0611784] [INSPIRE].

[120] F. De Bernardis, L. Pagano and A. Melchiorri, New constraints on the reheating temperature of the universe after WMAP-5, Astropart. Phys. 30 (2008) 192 [InSPIRE].

[121] P.F. de Salas, M. Lattanzi, G. Mangano, G. Miele, S. Pastor and O. Pisanti, Bounds on very low reheating scenarios after Planck, Phys. Rev. D 92 (2015) 123534 [arXiv: 1511.00672] [INSPIRE].

[122] M. Hufnagel, K. Schmidt-Hoberg and S. Wild, BBN constraints on MeV-scale dark sectors. Part II. Electromagnetic decays, JCAP 11 (2018) 032 [arXiv:1808.09324] [INSPIRE].

[123] T. Hasegawa, N. Hiroshima, K. Kohri, R.S.L. Hansen, T. Tram and S. Hannestad, $\mathrm{MeV}$-scale reheating temperature and thermalization of oscillating neutrinos by radiative and hadronic decays of massive particles, JCAP 12 (2019) 012 [arXiv: 1908.10189] [INSPIRE].

[124] M. Kawasaki, K. Kohri, T. Moroi, K. Murai and H. Murayama, Big-bang nucleosynthesis with sub-GeV massive decaying particles, JCAP 12 (2020) 048 [arXiv:2006.14803] [INSPIRE].

[125] P.F. Depta, M. Hufnagel and K. Schmidt-Hoberg, Updated BBN constraints on electromagnetic decays of MeV-scale particles, arXiv:2011.06519 [INSPIRE].

[126] J. Jaeckel and W. Yin, Boosted Neutrinos and Relativistic Dark Particles as Messengers from Reheating, JCAP 02 (2021) 044 [arXiv:2007.15006] [INSPIRE]. 